\title{
A Fusion Parameter Method for Classifying Freshness of Fish Based on Electrochemical Impedance Spectroscopy
}

\author{
Jian Sun, ${ }^{1}$ Yuhao Liu, ${ }^{1}$ Gangshan Wu, ${ }^{1}$ Yecheng Zhang $\left(\mathbb{D},{ }^{2}\right.$ Rongbiao Zhang, ${ }^{2}$ and X. J. Li ${ }^{3}{ }^{3}$ \\ ${ }^{1}$ School of Information Engineering, Jiangsu Vocational College of Agriculture and Forestry, Jurong 212499, China \\ ${ }^{2}$ School of Electrical and Information Engineering, Jiangsu University, Zhenjiang 212013, China \\ ${ }^{3}$ School of Mechanical Engineering, Jiangsu University, Zhenjiang 212013, China \\ Correspondence should be addressed to Yecheng Zhang; 15050859371@139.com and X. J. Li; xingjli@yeah.net
}

Received 9 November 2020; Revised 22 February 2021; Accepted 27 February 2021; Published 11 March 2021

Academic Editor: Daniel Cozzolino

Copyright (c) 2021 Jian Sun et al. This is an open access article distributed under the Creative Commons Attribution License, which permits unrestricted use, distribution, and reproduction in any medium, provided the original work is properly cited.

Compared with using a single characteristic parameter of electrochemical impedance spectroscopy (EIS) to classify the freshness of fish samples from different origins, more characteristic parameters could bring higher accuracy as well as complexity, subjectivity, and uncertainty. In order to eliminate the disadvantages of the multiparameter model, a data fusion method based on model similarity (DFMS) was proposed in this study. The similarity relation between the freshness models based on EIS characteristic parameters and physicochemical indicator was analyzed and quantified accordingly, and then, the weighting factors of the fusion model were determined. The classification accuracy rate of fish freshness based on DFMS was 9.2 15\% greater than that of a single EIS characteristic parameter. The novel dimensionless fusion parameter method proposed in this article might provide a simple yet effective indicator for EIS-based food quality evaluation.

\section{Introduction}

Freshness is an important quality attribute in the shelf life of freshwater fish, and the edible value reduces rapidly due to endogenous autolytic enzymes and microorganism after slaughter. The pathogenic microorganisms and microbial toxins produced in the process of spoilage pose a serious threat to the health of consumers [1]. Efficient, convenient, and real-time detection method of freshness plays an important role in the fields of food safety, quarantine of meat products, and agricultural products processing. Traditional freshness evaluation methods mainly include sensory assessment, physicochemical analysis, and microbiological experiment. As a rapid, nondestructive, and low-cost detection method, sensory assessment is widely used in daily life; however, the influence of personal subjective cannot be eliminated completely by this assessment, even for welltrained inspectors. Although physicochemical and microbiological methods can reach high detection accuracy, they require high professional skills of laboratory personnel, and the detection is destructive and time-consuming [2]. In the last two decades, different types of instrument detection technics, like electronic nose [3, 4], electronic tongue [5], near-infrared spectroscopy [6], dielectric spectra [7], hyperspectral imaging [8], and EIS [9-13], were developed to meet the demand of real-time detection in consumer market and online quality monitor in food processing factory [14].

Compared with the other instrument detection methods, EIS not only has advantages in low cost, rapid detection, simple pretreatment, and portable carrying, but also can provide information of internal organization of biological tissue [15] or somewhere difficult to reach by conventional means [16]. Thus, it is widely used in the field of agricultural engineering [17], food science [18], medical diagnosis [19], and battery technology $[20,21]$. For meat detection, EIS was applied to evaluate the taste and tenderness of beef [22], assess freshness of fish $[23,24]$, detect salt content of smoked products on production line [25-27], and real-time monitor the fat and moisture content during meat processing $[28,29]$. However, the body composition of fish varies with the feeding environment, fishing season, genetic genes, and so on. Grigorakis [30] reported that there was a 13-fold 
difference in body fat percentage between wild and cultured sea bream. The basic electric property would inevitably vary widely depending on the individual due to good insulation property of the body fat. The influence of individual differences on EIS detection results is one of the most important bottlenecks restricting the commercialization and the further development of bioimpedance technology. In the former research work, Kramers-Kronig's approach [11, 31-36] and Distribution of Relaxation Times (DRT) method [37-41] are effective tools to evaluate and process the mass electrochemical impedance data in certain fields.

The characteristic parameters of the impedance spectrum can be divided into two main categories, i.e., absolute coordinate parameter and morphological characteristic parameter. The former is closely related to the coordinates of the Bode diagram, such as modulus and phase angle; the latter is independent of the coordinate value but related to the morphology of the curve of impedance spectrum. In our previous study $[42,43]$, the morphological characteristic was chosen to detect the samples from different origins. The results show remarkable effect on improving the accuracy of detection and classification, because the morphological parameter was less affected by the difference of basic electrical properties of samples. With further analysis, we found that each characteristic parameter had its advantages, insufficiencies, and best application scope. Specifically, morphological characteristic parameter changed significantly near the critical point of spoilage, no matter the samples are from the same origin or different ones. However, the correlation between morphological characteristic parameter and physicochemical indicator in autolytic stage of spoilage was poor. The measuring points during this period constituted the main body of misjudgment set of morphological characteristic parameter. In contrast, the changing trend of absolute coordinate parameter was a high coincidence with that of physicochemical indicator during the whole storage period under laboratory conditions when samples come from the same origin, but it was very sensitive to individual differences in practical applications. Due to potential complementarities between the two characteristic parameters, parameter fusion technique may be an effective approach to improve the classification accuracy in practical application environment.

Parameter fusion technique has been employed in predicting capacities of lithium-ion battery [44, 45], estimating the freshness of food and other fields; however, the existing parameter fusion methods applicable to the field of food quality assessment have not yet proposed a standardized solution to the determination of weighting factors. This key point of parameter fusion method, such as global stability index (GSI) [46], was often implemented based on experience or semiexperience [47-49], which may lead to subjectivity and uncertainty.

To improve the classification accuracy of fish freshness in practical applications, a data fusion method based on model similarity (DFMS) was proposed to integrate the advantages of morphological characteristic parameter and absolute coordinate parameter from EIS experiment. The classification accuracy rate based on DFMS and single characteristic parameter were compared to verify the effect of fusion model. The innovative method proposed in this article not only improved the classification quality of EIS, but also provided a new solution to the quality evaluation of food.

\section{Materials and Methods}

2.1. Samples Preparation. To simulate the actual application scenario, 30 silver carp were purchased from 5 different retailers, 15 of which were used to determine the model parameters (group M), and the other 15 samples were used for model test (group T). Samples were delivered to the laboratory with oxygen supplement and killed by a thump on the head after filtrating anesthesia with ice water. Then scales and guts were removed. After cleaning, belly flesh was cut into $60 \times 30 \mathrm{~mm}$ pieces by a clean stainless-steel knife. The fillets were sealed with sterile polyethylene bag and placed in a $4^{\circ} \mathrm{C}$ refrigerator.

2.2. Physicochemical Analysis. The total volatile base nitrogen (TVB-N) is the protein decomposition product in the fish spoilage process. Semimicro fixation of nitrogen method was used to measure TVB-N value. After elimination of fishbone and sinew, $10 \mathrm{~g}$ of fish flesh was weighed and grinded into fish mud. Distilled water was added to $100 \mathrm{~mL}$, followed by stirring, soaking, and filtrating after $30 \mathrm{~min}$. $5 \mathrm{~mL}$ of filtrate drop was taken into a Kjeldahl apparatus (Huanya Experimental Instrument Co., Ltd., Shanghai, China); $5 \mathrm{ml}$ of $\mathrm{MgO}$ solution ( $10 \mathrm{~g} / \mathrm{L})$ subsequently was added quickly. TVB-N in the filtrate was distilled off with water vapor and collected by absorbent composed of boric acid solution $(20 \mathrm{~g} / \mathrm{L})$ and a mixed indicator prepared by adding $0.1 \mathrm{~g}$ of methyl red and $0.1 \mathrm{~g}$ of methylene blue into $100 \mathrm{~mL}$ of ethanol. Immediately after distillation, absorbent was titrated with a dilute hydrochloric acid solution $(0.01 \mathrm{~mol} / \mathrm{L})$. Subsequently, $5 \mathrm{~mL}$ of distilled water was used instead of the filtrate for the blank test. TVB-N was calculated as follows:

$$
X=\frac{\left(V_{1}-V_{2}\right) \times c \times 14}{m \times\left(V / V_{0}\right)} \times 100,
$$

where $X$ is the TVB-N value in terms of $\mathrm{mg} / 100 \mathrm{~g}$; $V_{1}$ is the consumption of hydrochloric acid solution in the experiment $(\mathrm{mL}) ; V_{2}$ is the consumption of hydrochloric acid solution in blank test $(\mathrm{mL}) ; c$ is the molar concentration of the hydrochloric acid solution in terms of $\mathrm{mol} / \mathrm{L} ; m$ is the measured mass of the flesh $(\mathrm{g})$; $V$ is the filtrate volume added into apparatus $(5 \mathrm{~mL})$; and $V_{0}$ is the total volume of the filtrate $(100 \mathrm{~mL})$.

TVB-N was measured every day within 8 days after slaughter. The final result was the average of two repeated measurements.

2.3. EIS Measurement. CHI660E electrochemical workstation (CH Instruments, Inc., Texas, USA) was used to measure EIS of fillets. The excitation voltage amplitude was set as $30 \mathrm{mV}$. 60 points with equal intervals were chosen on 
logarithmic coordinate axis between $10 \mathrm{~Hz}$ and $1 \mathrm{M} \mathrm{Hz}$ as the measurement frequency.

Two-electrode mode was used in measurement system. Two platinum $(\mathrm{Pt})$ wire electrodes with diameter of $0.5 \mathrm{~mm}$ were vertically inserted into the flesh. The depth and spacing between the two electrodes were both $10 \mathrm{~mm}$.

The frequency of EIS detection was the same as that of TVB-N measurement.

2.4. Data Analysis. DFMS was calculated by MATLAB software (version 7.10). Correlation analysis was used to extract characteristic parameters from absolute coordinate parameters. The accuracy rate of freshness classification was used to evaluate model effects based on parameter fusion and single parameter.

\section{Results and Discussion}

3.1. Change of $T V B-N$ during Storage. The average TVB-N values of group $M$ during storage are shown in Figure 1. TVB-N increased monotonously as storage time increased. In the first 3 days, the change of TVB-N was gentle. The rate of increase began to speed up on the 4 th day. According to the Chinese National Standard GB/T 2009.45-2003, the rejection limit for TVB-N in freshwater fish $(20 \mathrm{mg} / 100 \mathrm{~g})$ was reached on the 6th day.

Autolytic enzymes and microorganisms were two main factors responsible for protein decomposition in spoilage process [50]. At the early stage of storage, microorganisms needed time to adapt to the new environment, autolytic enzymes played a leading role in TVB-N production, and the corresponding change of TVB-N was moderate. During the middle and late stages of storage, microorganisms gradually took the place of autolytic enzymes and became the main cause of protein decomposition due to the reproduction of dominant spoilage bacteria. The corresponding trend of change was rapid rise of TVB-N with the storage time.

3.2. Characteristic Parameter Extraction. Absolute coordinate parameters, like impedance modulus, phase angle, real part, and imaginary part, are closely related to the coordinate value in plane of EIS curve. The measurement result of CHI660E is expressed in the form of complex number composed of a real part and an imaginary part. However, impedance modulus and phase angle, which represent the reduction and skewing effect on electric current, have clearer physical meanings. Impedance modulus and phase angle are calculated as follows:

$$
\begin{aligned}
|Z| & =\sqrt{Z_{\mathrm{re}}^{2}+Z_{\mathrm{im}}^{2}}, \\
\theta & =-\operatorname{arctg} \frac{Z_{\mathrm{im}}}{Z_{\mathrm{re}}} \times \frac{180}{\pi},
\end{aligned}
$$

where $|Z|$ is impedance modulus in terms of $\Omega ; \theta$ is phase angle in terms of degree; and $Z_{\mathrm{re}}$ and $Z_{\mathrm{im}}$ are real and imaginary parts of impedance, respectively. In order to match the change trend of the impedance modulus and the
$\mathrm{PD}$, the phase angle was converted to positive number by the inverse operation.

Figure 2 is a Bode diagram showing the trends of the impedance modulus and phase angle in excitation frequency domain, where the abscissa is the measurement frequency, and the ordinates are the impedance modulus and phase angle, respectively.

The impedance modulus and phase angle varied with the frequency. Due to the capacitance caused by cell membrane and electric double layer on the electrode surface, the impedance modulus was more than $2000 \Omega$, and the phase angle was more than $40^{\circ}$ in low-frequency region. With the increase of the excitation frequency, the value of capacitance mentioned above decreased. Meanwhile, the impedance modulus dropped below $1000 \Omega$, and the phase angle dropped to the interval between $10^{\circ}$ and $30^{\circ}$.

The trend of impedance modulus curve changed obviously at about $100 \mathrm{~Hz}$. In the frequency range above $100 \mathrm{~Hz}$, the impedance modulus decreased slowly with the increase of frequency. In the low-frequency interval between 10 and $100 \mathrm{~Hz}$, the impedance modulus changed significantly. In this narrow frequency domain, the modulus value dropped rapidly from $7000 \Omega$ to $2000 \Omega$. The rapidly changing trend in low-frequency range was coincident with the frequency response of the electric double layer capacitance which was connected in series in the measurement circuit. Thus, the electrical property of fish was obscured by the capacitance of electric double layer below $100 \mathrm{~Hz}$. The effective frequency range reflecting the electrical property of fish was 100 to $1 \mathrm{M} \mathrm{Hz}$. In this gradual interval, there was a certain linear correlation between the absolute coordinate parameter corresponding to different frequencies; therefore, only one value at a specific frequency was chosen as the fusion parameter for the impedance modulus and phase angle, respectively.

As shown in Figure 3, the coefficients of determination between absolute coordinate parameters and TVB-N were obtained by correlation analysis method, and the biggest coefficient of determination with TVB-N value was selected as fusion parameter.

The abscissa is the excitation frequency, while the ordinate is the determination coefficient $\left(R^{2}\right)$ between the absolute coordinate parameter and TVB-N; the red bar represents phase angle, and the blue bar is impedance modulus. To simulate the actual application environment, the samples were randomly purchased from multiple retailers. Due to the individual differences of samples from different origins, the results of correlation analysis were lower than those of similar studies with samples from the same origin and the same batch.

The curve of determination coefficient between phase angle and TVB-N had two peaks in the frequency interval between 100 and $1 \mathrm{M} \mathrm{Hz}$, where one located at about $300 \mathrm{~Hz}$ and the other was in the domain from $80 \mathrm{k}$ to $400 \mathrm{k} \mathrm{Hz}$. The maximum $R^{2}$ was 0.60 , and the corresponding frequency of that was $100 \mathrm{kHz}$. The maximum $R^{2}$ of the impedance modulus appeared at $1 \mathrm{kHz}$ which was in the valley region of the phase angle; the value was 0.56. Therefore, the phase 
angle at $100 \mathrm{kHz}$ and the modulus at $1 \mathrm{k} \mathrm{Hz}$ were chosen as the absolute coordinate parameters for fusion modeling.

Contrary to absolute coordinate parameter, morphological characteristic parameter is independent of the coordinate value in plane of EIS curve and only relates to the shape of the curve. Common morphological characteristic parameters include slope, radians, and pole difference. Pole difference $(\mathrm{PD})$ was used in this study to participate in the modeling process. As shown in Figure 4, PD was the $y$-axis projection distance between the highest point and the lowest point in phase angle curve.

The change of PD during the storage time is shown in Figure 5. There was a transient rise in PD during the period of pre- and post-rigor mortis stage shortly after slaughter (stage 1). In autolytic stage, PD decreased slowly with the prolongation of storage time (stage 2). After the 6th day when the critical point of spoilage was reached, the decline rate of $\mathrm{PD}$ accelerated significantly (stage 3 ).

$\mathrm{PD}$ is the projection distance on the $y$-axis between the highest point and the lowest point of "S" shape part in the phase angle curve (Figure 1). According to the electrical principle, the phase angle curve of pure resistive element is a line segment on the $x$-axis and the PD value of that is 0 ; the $\mathrm{PD}$ values of the combined elements composed of resistances and capacitances are always greater than 0 . In the middle and late stages of spoilage, the cell membrane ruptured. The electrolyte of intracellular fluid outflowed and then fused with the extracellular fluid. The macroscopic electrical property of fish changed from resistance-capacitance mixing to pure resistance, and the corresponding $\mathrm{PD}$ value also tended to 0 .

\subsection{Data Fusion Method Based on Model Similarity (DFMS).}

The determination of weighting factor is one of the most important steps of fusion algorithm, but there is no specification method to obtain the optimal combination of weighting factors so far. Unlike extraction of absolute coordinate parameters, there is no direct linear relationship between the absolute coordinate parameter and morphological characteristic parameter in terms of mechanism. Thus, if correlation analysis is used to determine the weighting factor, a large sample size is required to ensure the validity of the results. In addition, the weighting factor can also be estimated through experience, but there is inevitably a certain degree of subjectivity and uncertainty.

Aimed at this problem, a data fusion method based on model similarity (DFMS) was proposed in this study. The models describing fish freshness, whether based on TVB-N or EIS characteristic parameters, all present as the curves varying with storage time. The core idea of this method is to compare and quantify the morphological differences between the curves of fusion parameters and control parameters and set a larger weighting coefficient for the fusion parameter which has smaller morphological differences with the curve of control parameter. The detailed calculation steps are as follows:

\section{(1) Curve Syntropy}

Most indicators related to food freshness have a general upward or downward trend with storage time. The impedance modulus, phase angle, and PD all monotonically decreased with time since the second day after slaughter. However, the TVB-N, as the control parameter, increased with time in all over the storage period. In order to ensure that the fusion parameters and control parameters had the same trend time, opposite numbers of impedance modulus, phase angle, and PD were calculated as fusion parameters. Thus, fusion parameters included impedance modulus $\left(M=m_{2}, m_{3}, \ldots, m_{8}\right)$, phase angle $\left(A=a_{2}, a_{3}, \ldots, a_{8}\right)$, and $\mathrm{PD}\left(P=p_{2}, p_{3}\right.$, $\left.\ldots, p_{8}\right)$, and the control parameter was TVB-N $\left(T=t_{2}, t_{3}, \ldots, t_{8}\right)$. Where $t_{\mathrm{i}}$ was the average values of phase angle and TVB-N in each measurement day for group $M$, and $m_{\mathrm{i}}, a_{\mathrm{i}}$, and $p_{\mathrm{i}}$ were the opposite numbers of corresponding values for modulus, phase angle, and PD.

\section{(2) Nondimensionalization}

Different parameters were not comparable due to different dimensions. Mean value method was used to nondimensionalize the data. The dimensionless impedance modulus $\left(M^{*}\right)$ was calculated as follows. $A^{*}, P^{*}$, and $T^{*}$ were calculated accordingly.

$$
m_{i}^{\prime}=\frac{m_{i}}{\left|\sum_{i=a}^{b} m_{i} /(b-a+1)\right|},
$$

where $a$ equals 2 and $b$ equals 8 representing the storage day.

\section{(3) Alignment of Initial Points}

Initial points of the curves were aligned though subtracting respective initial values.

$$
m_{i}^{*}=m_{i}^{\prime}-m_{a}^{\prime},
$$

where $m_{a}^{\prime}$ was the impedance modulus after nondimensionalization in the second day; the corresponding values of phase angle $\left(a_{i}^{*}\right)$ and $\mathrm{PD}\left(p_{i}^{*}\right)$ were calculated accordingly. The initial points of all curves were moved to coordinate origin.

(4) Calculation of Average Root Mean Squared Distance (RMSD)

$$
\overline{\operatorname{RMSD}}=\frac{\sqrt{\left(\sum_{i=a}^{b}\left(t_{i}^{*}-m_{j}^{*}\right)^{2}+\sum_{j=a}^{b}\left(t_{j}^{*}-a_{j}^{*}\right)^{2}+\sum_{k=a}^{b}\left(t_{k}^{*}-p_{k}^{*}\right)^{2}\right)}}{3(b-a+1)} .
$$


Average RMSD quantified the average morphological difference between the curves of fusion parameters and control parameter.
(5) Calculation of Minimum RMSD

$$
\operatorname{RMSD}_{\min }=\min \left(\min _{i} \sqrt{\left(t_{i}^{*}-m_{i}^{*}\right)^{2}}, \min _{j} \sqrt{\left(t_{j}^{*}-a_{j}^{*}\right)^{2}}, \min _{k} \sqrt{\left(t_{k}^{*}-p_{k}^{*}\right)^{2}}\right) .
$$

Minimum RMSD represented the minimum morphological difference between the curves of fusion parameters and control parameter.

(6) Calculation of RMSD of Fusion Parameters

$$
\operatorname{RMSD}_{M}=\sqrt{\frac{\sum_{i=a}^{b}\left(t_{i}^{*}-m_{i}^{*}\right)^{2}}{(b-a+1)}} .
$$

RMSD $_{M}$ was RMSD between curves of modulus and TVB-N; the corresponding values of phase angle $\left(\mathrm{RMSD}_{\mathrm{A}}\right)$ and PD $\left(\mathrm{RMSD}_{\mathrm{P}}\right)$ were calculated accordingly.

(7) Calculation of Morphological Similarity of Curve (MSC)

$$
\mathrm{MSC}_{M}=\frac{\left(\mathrm{RMSD}_{\min }+\overline{\mathrm{RMSD}}\right)}{\left(\mathrm{RMSD}_{M}+\overline{\mathrm{RMSD}}\right)}
$$

$\mathrm{MSC}_{\mathrm{M}}$ represented the morphological similarity between curves of modulus and TVB-N. Compared with the curves of all fusion parameters, the corresponding values of phase angle $\left(\mathrm{MSC}_{\mathrm{A}}\right)$ and $\mathrm{PD}$ $\left(\mathrm{MSC}_{\mathrm{P}}\right)$ were calculated accordingly.

(8) Calculation of Weighting Factors

Weighting factors of impedance modulus in equation (3) were obtained as follows; the corresponding values of phase angle $\left(\alpha_{A}\right)$ and $\operatorname{PD}\left(\alpha_{P}\right)$ were calculated accordingly.

$$
\alpha_{M}=\frac{\mathrm{MSC}_{M}}{\left(\mathrm{MSC}_{M}+\mathrm{MSC}_{A}+\mathrm{MSC}_{P}\right)} .
$$

\section{(9) Normalization of Fusion Parameters}

Normalization of fusion parameters was implemented according to the equation in GSI method [30]. The variation term $V_{i j}$ was calculated as follows:

$$
V_{i j}=\frac{C_{i j}-C_{i 0}}{L_{i}-C_{i 0}}
$$

where $C_{i j}$ is the measured value of indicator $i$ at time $j$ units; $C_{i 0}$ is the initial value of indicator $i$; and $L_{i}$ is the threshold value of indicator $i . V_{i j}$ describes the measured variation as compared to the maximum tolerated variation of indicator $i$ at time $j$ units.

(10) Weighted Fusion

The value of DFMS indicator at $j$ time is calculated as follows:

$$
\operatorname{DFMS}_{j}=\sum_{1}^{n} \alpha_{i} V_{i j}
$$

where $n$ is the number of indicators involved in the model; $V_{i j}$ is the variation terms; and $\alpha_{i}$ is the weighting factor of the indicator $i$.

3.4. Freshness Classification and Prediction Based on DFMS. EIS measurement results of group $M$ were processed though DFMS. Calculation result of the weighting factors of fusion parameters are, respectively, as follows: modulus is 0.327 , phase angle is 0.296 , and PD is 0.377 . And the freshness classification and prediction based on DFMS vs. storage time are, respectively, shown in Figures 6(a) and 6(b).

Contrary to TVB-N, DFMS indicator presented a monotonous downward trend with storage time. Compared with Figure 1, TVB-N reached the rejection limit on the 6th day; the corresponding DFMS indicator value was 0.47 in Figure 6(a). At the corresponding storage time, the values of modulus, phase angle, and PD were 1120, 14.8, and 6.4, respectively. Those values were considered as critical points of spoilage for each characteristic parameter. The modulus, phase angle, PD, and DFMS indicator all decreased with time. The freshness classification results of those were judged to be correct when the measurement values between 1st and 5 th days were greater than critical points, or the measurement values between 6th and 8th days were less than critical points. Moreover, a prediction based on DFMS launched by another independent experiment is shown in Figure 6(b). The accuracy rates of freshness determination based on DFMS indicator and single indicators are shown in Table 1.

The calibration samples (group $M$ ) were used to filter the characteristic parameters and calculate the weighting factors in fusion model, and the test samples (group T) did not participate in the modeling process. The latter could more 


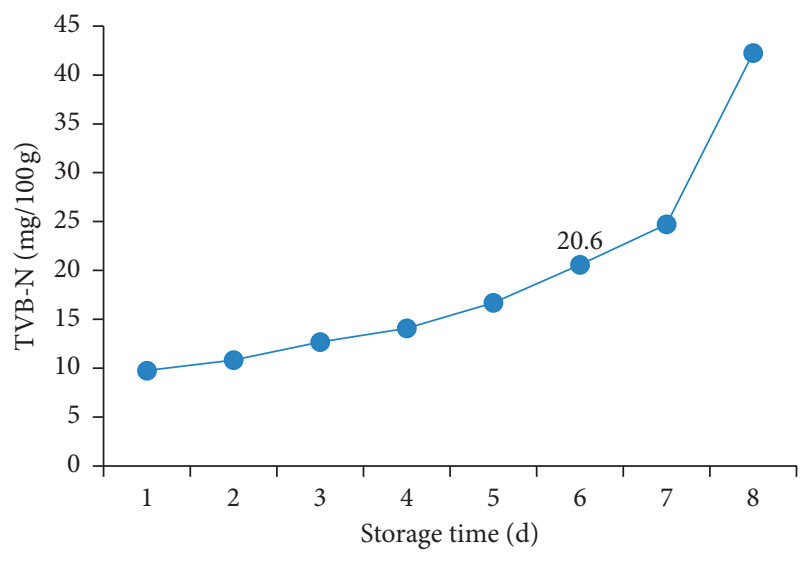

FIgURE 1: TVB-N values during the storage time.

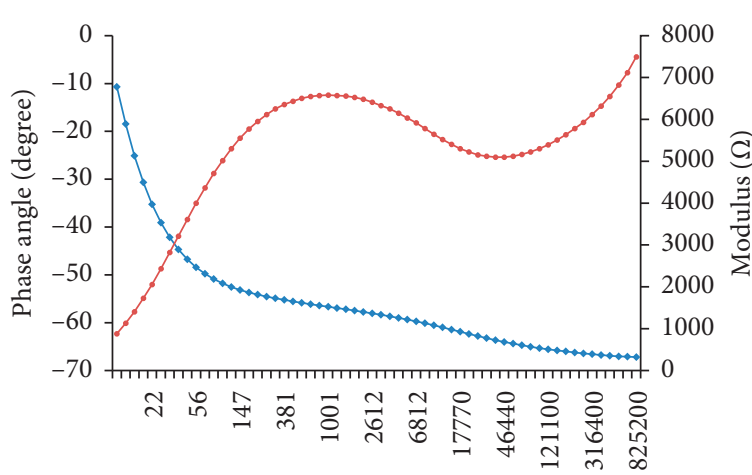

Frequency $(\mathrm{Hz})$

$\rightarrow$ Modulus

$\longrightarrow$ Phase angle

Figure 2: Bode diagram.

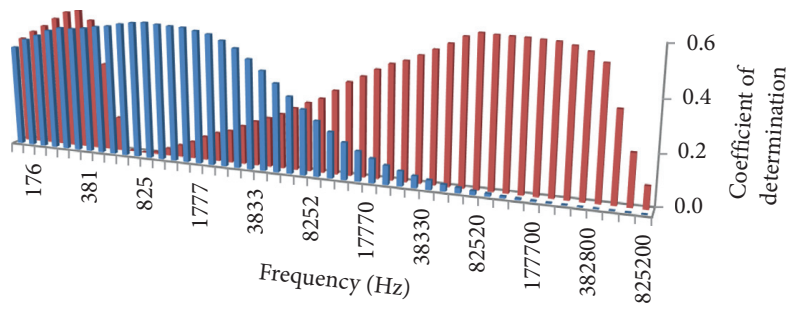

Modulus

Phase angle

FIgURE 3: Determination coefficients between absolute coordinate parameters and TVB-N at different frequencies.

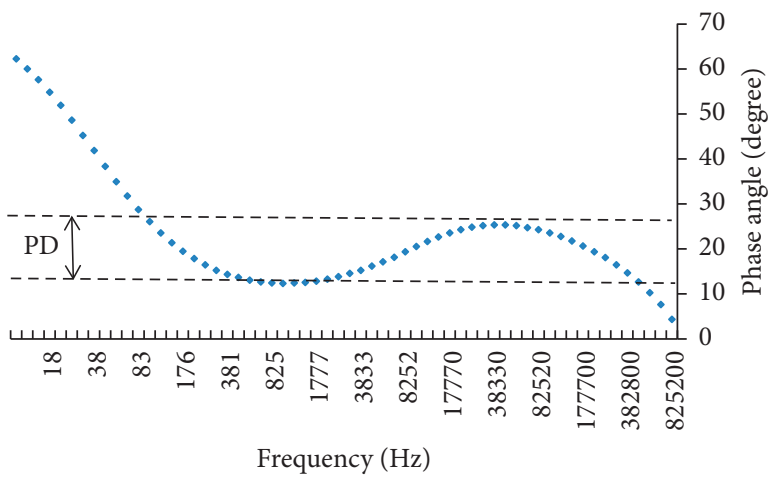

FIgURE 4: Extraction of the pole difference parameter.

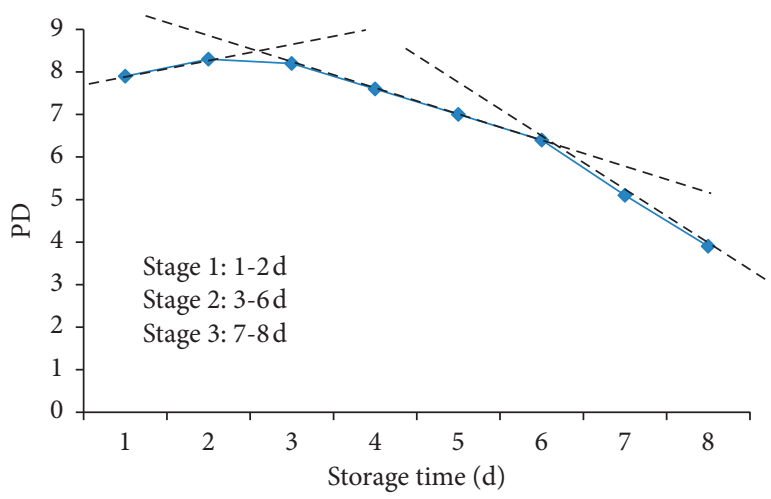

Figure 5: Pole difference values during storage time.

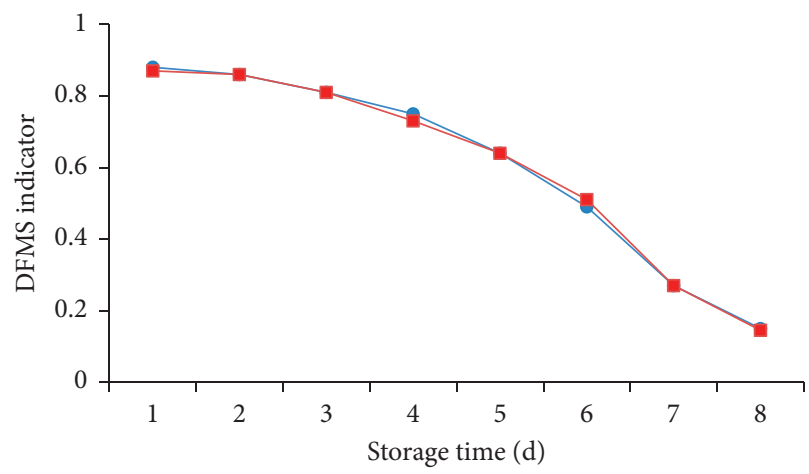

(a)

(b)

FIGURE 6: DFMS indicator during storage time. 
TABLE 1: Accuracy rate of freshness determination of different quality indicators.

\begin{tabular}{lcccc}
\hline Group & Modulus (\%) & Phase angle (\%) & PD (\%) & DFMS indicator (\%) \\
\hline $\mathrm{M}$ & 82.5 & 85.8 & 86.7 & 95.0 \\
$\mathrm{~T}$ & 79.2 & 83.3 & 85.0 & 94.2 \\
\hline
\end{tabular}

effectively reflect the classification effect under the actual application scenario. For group $T$, the classification accuracy rate of DFMS indicator was $94.2 \%$, significantly higher than that of modulus (79.2\%), phase angle (83.3\%), and PD (85.0\%).

\section{Conclusions}

In order to improve the freshness classification accuracy of samples from multiple origins, impedance modulus, phase angle, and PD were fused by DFMS. Compared with the single characteristic parameter, the freshness classification accuracy rate based on fusion model increased from $79.2 \%-85.0 \%$ to $94.2 \%$. The results indicated that DFMS can effectively distribute weighting coefficient in the key step of fusion model. The innovative method proposed in this article not only improved the classification quality of EIS in practical application environment, but also provided a new idea on the quality evaluation of food which could eradicate subjectivity and uncertainty from classification results. EIS freshness classification method based on parameter fusion could be a potential solution to the development of portable detection device in consumer market and online monitor system in food processing factory.

\section{Abbreviations}

EIS: $\quad$ Electrochemical impedance spectroscopy

DFMS: Data fusion method based on model similarity

GSI: Global stability index

Group Modeling group (the samples which were used to

$M$ : $\quad$ determine the model parameters)

Group Testing group (the samples which were used for $T: \quad$ model test)

TVB-N: Total volatile base nitrogen

PD: $\quad$ Pole difference (morphological parameters of impedance spectroscopy)

RMSD: Root mean squared distance (process parameters in DFMS calculation)

MSC: $\quad$ Morphological similarity of curve (process parameters in DFMS calculation).

\section{Data Availability}

The data used to support the findings of this study are included within the article.

\section{Conflicts of Interest}

The authors declare that there are no conflicts of interest regarding the publication of this paper.

\section{Acknowledgments}

This work was financially supported by the National Natural Science Foundation of China (Grant no. 61673195) and Youth Support Project of Jiangsu Vocational College of Agriculture and Forestry (Grant no. 2020kj013).

\section{References}

[1] A. E. Ghaly, D. Dave, S. Budge, and M. S. Brooks, "Fish spoilage mechanisms and preservation techniques: review," American Journal of Applied Sciences, vol. 7, no. 7, pp. 859877, 2010.

[2] J.-H. Cheng, D.-W. Sun, X.-A. Zeng, and D. Liu, "Recent advances in methods and techniques for freshness quality determination and evaluation of fish and fish fillets: a review," Critical Reviews in Food Science and Nutrition, vol. 55, no. 7, pp. 1012-1225, 2015.

[3] M. O’Connell, G. Valdora, G. Peltzer, and R. M. Negri, “A practical approach for fish freshness determinations using a portable electronic nose," Sensors \& Actuators, B: Chemical, vol. 80, pp. 149-154, 2001.

[4] M. Li, H. Wang, L. Sun, G. Zhao, and X. Huang, "Application of electronic nose for measuring total volatile basic nitrogen and total viable counts in packaged pork during refrigerated storage," Journal of Food Science, vol. 81, no. 4, pp. M906-M912, 2016.

[5] J. Han, L. Huang, Z. Gu, and S. Deng, "Evaluation of fish quality and freshness based on the electronic tongue," Transactions of the Chinese Society of Agricultural Engineering, vol. 2008, 2008.

[6] S. Kuroki, T. Kanoo, H. Itoh, Y. Ohkawa, and H. Kamisoyama, "Nondestructive measurement of yolk viscosity in lightly heated chicken shell eggs," Journal of Food Engineering, vol. 205, pp. 18-24, 2017.

[7] M. Castro-Giráldez, P. Botella, F. Toldrá, and P. Fito, "Lowfrequency dielectric spectrum to determine pork meat quality," Innovative Food Science \& Emerging Technologies, vol. 11, no. 2, pp. 376-386, 2010.

[8] D. Fu and Q. Wang, "Predictive models for the detection of egg freshness, acidity and viscosity using hyper-spectral imaging," Food Science, vol. 37, no. 22, pp. 173-179, 2016.

[9] M. E. Orazem and B. Tribollet, 16. Methods for Representing Impedance, John Wiley \& Sons, Inc., Hoboken, NJ, USA, 2008.

[10] M. E. Orazem, N. Pébère, and B. Tribollet, "Enhanced graphical representation of electrochemical impedance data," Journal of the Electrochemical Society, vol. 153, no. 4, 2006.

[11] P. Agarwal, "Application of measurement models to impedance spectroscopy III. Evaluation of consistency with the kramers-kronig relations," Journal of The Electrochemical Society, vol. 142, no. 12, p. 4149, 1995.

[12] F. Ciucci, "Modeling electrochemical impedance spectroscopy," Current Opinion in Electrochemistry, vol. 13, pp. 132139, 2019.

[13] E. Ivers-Tiffée, A. Weber, and H. Schichlein, "Electrochemical impedance spectroscopy," in Handbook of Fuel Cells, 
W. Vielstich, A. Lamm, H. A. Gasteiger, and H. Yokokawa, Eds., Wiley, Hoboken, NJ, USA, 2010.

[14] Y. H. Zhang and Y. Meng, "Research and progress of nondestructive testing technology of meat quality," Science \& Technology of Food Industry, vol. 33, pp. 392-391, 2012.

[15] S. M. M. Islam, M. A. R. Reza, and M. A. Kiber, "Development of multi-frequency electrical impedance spectroscopy (EIS) system for early detection of breast cancer," International Journal of Electronics \& Informatics, vol. 2, pp. 26-32, 2013.

[16] T. Repo, J. Laukkanen, and R. Silvennoinen, "Measurement of the tree root growth using electrical impedance spectroscopy," Silva Fennica, vol. 39, pp. 159-166, 2005.

[17] T. Repo, A. Korhonen, T. Lehto, and R. Silvennoinen, “Assessment of frost damage in mycorrhizal and non-mycorrhizal roots of Scots pine seedlings using classification analysis of their electrical impedance spectra," Trees, vol. 30, no. 2, pp. 483-495, 2016.

[18] G. Chee, N. Rungraeng, J. H. Han, and S. Jun, "Electrochemical impedance spectroscopy as an alternative to determine dielectric constant of potatoes at various moisture contents," Journal of Food Science, vol. 79, no. 2, pp. E195-E201, 2014.

[19] U. G. Kyle, I. Bosaeus, A. D. De Lorenzo et al., "Bioelectrical impedance analysis-part II: utilization in clinical practice," Clinical Nutrition, vol. 23, no. 6, pp. 1430-1453, 2004.

[20] M. Papac, V. Stevanovi, A. Zakutayev et al., "Triple ionic-electronic conducting oxides for next-generation electrochemical devices," Nature Materials, vol. 20, no. 3, pp. 301-313, 2020.

[21] S. Randau, D. A. Weber, O. Kötz et al., "Benchmarking the performance of all-solid-state lithium batteries," Nature Energy, vol. 5, no. 3, pp. 259-270, 2020.

[22] J. Lepetit, P. Salé, R. Favier, and R. Dalle, "Electrical impedance and tenderisation in bovine meat," Meat Science, vol. 60, no. 1, pp. 51-62, 2002.

[23] E. Pérez-Esteve, A. Fuentes, R. Grau et al., "Use of impedance spectroscopy for predicting freshness of sea bream (Sparus aurata)," Food Control, vol. 35, no. 1, pp. 360-365, 2014.

[24] Ø. G. Martinsen, S. Grimnes, and P. Mirtaheri, "Non-invasive measurements of post-mortem changes in dielectric properties of haddock muscle-a pilot study," Journal of Food Engineering, vol. 43, no. 3, pp. 189-192, 2000.

[25] A. Rizo, A. Fuentes, I. Fernández-Segovia, R. Masot, M. Alcañiz, and J. M. Barat, "Development of a new salmon salting-smoking method and process monitoring by impedance spectroscopy," LWT-Food Science and Technology, vol. 51, no. 1, pp. 218-224, 2013.

[26] P. Karásková, A. Fuentes, I. Fernández-Segovia, M. Alcañiz, R. Masot, and J. M. Barat, "Development of a low-cost nondestructive system for measuring moisture and salt content in smoked fish products," Procedia Food Science, vol. 1, pp. 1195-1201, 2011.

[27] R. Masot, M. Alcañiz, A. Fuentes et al., "Design of a low-cost non-destructive system for punctual measurements of salt levels in food products using impedance spectroscopy," Sensors and Actuators A: Physical, vol. 158, no. 2, pp. 217-223, 2010.

[28] M. J. Marchello, W. D. Slanger, and J. K. Carlson, "Bioelectrical impedance: fat content of beef and pork from different size grinds," Journal of Animal Science, vol. 77, no. 9, pp. 2464-2468, 1999.

[29] M. Chanet, C. Rivière, and P. Eynard, "Electric impedance spectrometry for the control of manufacturing process of comminuted meat products," Journal of Food Engineering, vol. 42, no. 3, pp. 153-159, 1999.

[30] K. Grigorakis, "Compositional and organoleptic quality of farmed and wild gilthead sea bream (Sparus aurata) and sea bass (Dicentrarchus labrax) and factors affecting it: a review," Aquaculture, vol. 272, no. 1-4, pp. 55-75, 2007.

[31] B. A. Boukamp, "A linear kronig-kramers transform test for immittance data validation," Journal of The Electrochemical Society, vol. 142, no. 6, pp. 1885-1894, 1995.

[32] B. A. Boukamp and J. R. Macdonald, "Alternatives to KronigKramers transformation and testing, and estimation of distributions," Solid State Ionics, vol. 74, no. 1-2, pp. 85-101, 1994.

[33] J. M. Esteban and M. E. Orazem, "On the application of the kramers-kronig relations to evaluate the consistency of electrochemical impedance data," Journal of the Electrochemical Society, vol. 138, no. 1, pp. 67-76, 1991.

[34] B. Hirschorn and M. E. Orazem, "On the sensitivity of the kramers-kronig relations to nonlinear effects in impedance measurements," Journal of the Electrochemical Society, vol. 156, no. 10, pp. C345-C351, 2009.

[35] J. Liu, T. Hei Wan, and F. Ciucci, "A Bayesian view on the Hilbert transform and the Kramers-Kronig transform of electrochemical impedance data: probabilistic estimates and quality scores," Electrochimica Acta, vol. 357, Article ID 136864, 2020.

[36] F. Ciucci, "The Gaussian process hilbert transform (GP-HT): testing the consistency of electrochemical impedance spectroscopy data," Journal of the Electrochemical Society, vol. 167, no. 12, Article ID 126503, 2020.

[37] I.-T. Ellen, E. Ellen, and A. Weber, "Evaluation of electrochemical impedance spectra by the distribution of relaxation times," Journal-Ceramic Society Japan, vol. 125, no. 4, pp. 193-201, 2017.

[38] J. Liu and F. Ciucci, "The Gaussian process distribution of relaxation times: a machine learning tool for the analysis and prediction of electrochemical impedance spectroscopy data," Electrochimica Acta, vol. 331, Article ID 135316, 2019.

[39] T. Hei Wan, M. Saccoccio, C. Chen, and F. Ciucci, "Influence of the discretization methods on the distribution of relaxation times deconvolution: implementing radial basis functions with DRTtools," Electrochimica Acta, vol. 184, pp. 483-499, 2015.

[40] D. Clematis, S. Presto, M. P. Carpanese et al., "Distribution of relaxation times and equivalent circuits analysis of Ba0.5Sr0.5Co0.8Fe0.2O3- $\delta$," Catalysts, vol. 9, no. 5, p. 441, 2019.

[41] J. Huang, M. Papac, and R. O’Hayre, "Towards robust autonomous impedance spectroscopy analysis: a calibrated hierarchical Bayesian approach for electrochemical impedance spectroscopy (EIS) inversion," Electrochimica Acta, vol. 367, Article ID 137493, 2020.

[42] J. Sun, R. Zhang, Y. Zhang, G. Li, and Q. Liang, "Estimating freshness of carp based on EIS morphological characteristic," Journal of Food Engineering, vol. 193, pp. 58-67, 2017.

[43] J. Sun, R. Zhang, Y. Zhang et al., "Classifying fish freshness according to the relationship between EIS parameters and spoilage stages," Journal of Food Engineering, vol. 219, pp. 101-110, 2018.

[44] K. Liu, Y. Shang, Q. Ouyang et al., "A data-driven approach with uncertainty quantification for predicting future capacities and remaining useful life of lithium-ion battery," IEEE Transactions on Industrial Electronics, vol. 68, no. 4, p. 1, 2020. 
[45] K. Liu, X. Hu, Z. Wei et al., "Modified Gaussian process regression models for cyclic capacity prediction of lithium-ion batteries," IEEE Transactions on Transportation Electrification, vol. 5, no. 4, pp. 1225-1236, 2020.

[46] H. Hong, Y. Luo, S. Zhu, and H. Shen, "Application of the general stability index method to predict quality deterioration in bighead carp (Aristichthys nobilis) heads during storage at different temperatures," Journal of Food Engineering, vol. 113, no. 4, pp. 554-558, 2012.

[47] S. Zhu, Y. Luo, L. Feng, and Y. Bao, "Establishment of kinetic models based on electrical conductivity and global stability index for predicting the quality of allogynogenetic crucian carps (C arassius auratus gibelio) during chilling storage," Journal of Food Processing and Preservation, vol. 39, no. 2, pp. 167-174, 2015.

[48] Y. L. Bao, Y. K. Luo, Y. M. Zhang, Y. F. Shen, and H. X. Shen, "Application of the global stability index method to predict the quality deterioration of blunt-snout bream (Megalobrama amblycephala) during chilled storage," Food Science and Biotechnology, vol. 22, pp. 1309-1313, 2013.

[49] M. Achour, "A new method to assess the quality degradation of food products during storage," Journal of Food Engineering, vol. 75, no. 4, pp. 560-564, 2006.

[50] M. C. Pina-Pérez, A. Rivas, A. Martínez, and D. Rodrigo, "Antimicrobial potential of macro and microalgae against pathogenic and spoilage microorganisms in food," Food Chemistry, vol. 235, pp. 34-44, 2017. 